\title{
Pravastatin Reduces the Risk of Atherothrombotic Stroke when Administered within Six Months of an Initial Stroke Event
}

\author{
Naohisa Hosomi ${ }^{1}$, Yoji Nagai ${ }^{2}$, Kazuo Kitagawa ${ }^{3}$, Yoko Nakagawa ${ }^{4}$, Shiro Aoki ${ }^{1}$, Tomohisa Nezu ${ }^{1}$, \\ Tatsuo Kagimura ${ }^{4}$, Hirofumi Maruyama ${ }^{1}$, Hideki Origasa ${ }^{5}$, Kazuo Minematsu ${ }^{6}$, Shinichiro Uchiyama ${ }^{7}$ and \\ Masayasu Matsumoto ${ }^{1,8}$; J-STARS collaborators
}

${ }^{1}$ Department of Clinical Neuroscience and Therapeutics, Hiroshima University Graduate School of Biomedical and Health Sciences, Hiroshima, Japan

${ }^{2}$ Center for Clinical Research, Kobe University Hospital, Kobe, Japan

${ }^{3}$ Department of Neurology, Tokyo Women's Medical University School of Medicine, Tokyo, Japan

${ }^{4}$ Division of Medical Statistics, Translational Research Informatics Center, Foundation for Biomedical Research and Innovation, Kobe, Japan

${ }^{5}$ Division of Biostatistics and Clinical Epidemiology, University of Toyama Graduate School of Medicine and Pharmaceutical Sciences, Toyama, Japan

${ }^{6}$ National Cerebral and Cardiovascular Center, Suita, Japan

${ }^{7}$ International University of Health and Welfare, Center for Brain and Cerebral Vessels, Sanno Hospital and Sanno Medical Center, Tokyo, Japan

${ }^{8}$ Hoshigaoka Medical Center, Japan Community Healthcare Organization (JCHO), Osaka, Japan

\begin{abstract}
Aims: The J-STARS study examined whether pravastatin $(10 \mathrm{mg} /$ day) reduces recurrence of stroke in non-cardioembolic ischemic stroke patients who were enrolled within 1 month to 3 years after initial stroke events (ClinicalTrials.gov, NCT00221104). The main results showed that the frequency of atherothrombotic stroke was low in pravastatin-treated patients, although no effect of pravastatin was found for the other stroke subtypes. We evaluated differences of early (within 6 months) or late (after 6 months) pravastatin treatment benefits on the incidence of stroke or transient ischemic attack (TIA), as well as atherothrombotic stroke and the other subtypes.

Methods: Subjects in the J-STARS study were classified into two cohorts, depending on whether they enrolled early ( 1 to 6 months) or late ( 6 months to 3 years) following initial stroke events.

Results: A total of 1578 patients (491 female, $66.2 \pm 8.5$ years) were randomly assigned to either the pravastatin group $(n=793 ; n=426$ in the early cohort, $n=367$ in the late cohort) or the control group ( $n=785 ; n=417$ in the early cohort, $n=368$ in the late cohort). During the follow-up of $4.9 \pm$ 1.4 years, the rate of atherothrombotic stroke was lower in the pravastatin group compared to controls in the early cohort $(0.24$ vs. $0.88 \% /$ year, $p=0.01)$ but not in the late cohort $(0.17$ vs. $0.39 \% /$ year, $p=0.29$ ). However, this difference of pravastatin effect on atherothrombotic stroke was not significantly interacted by the early or late cohort $(p=0.59)$. The incidence rates of other stroke subtype were not different in between pravastatin and control groups despite the timing of entry.

Conclusions: Pravastatin is likely to reduce atherothrombotic stroke in patients enrolled within 6 months after stroke onset. However, the clinical efficacy for prevention of recurrent stroke was not conclusive with earlier statin treatment.
\end{abstract}

Key words: Statin, Stroke, Prevention, Atherothrombotic, Cholesterol

\section{Introduction}

Stroke is a heterogeneous disease with different etiologies, and may present with or without underlying arterial pathologies ${ }^{1-5)}$. Thus, the benefits of statins may be different depending on the stroke subtype. The Japan Statin Treatment Against Recurrent Stroke (J-STARS) study was conducted to examine whether pravastatin $(10 \mathrm{mg} /$ day $)$ reduces recurrence of stroke in non-cardioembolic ischemic stroke patients. The 
Table 1. Baseline characteristics

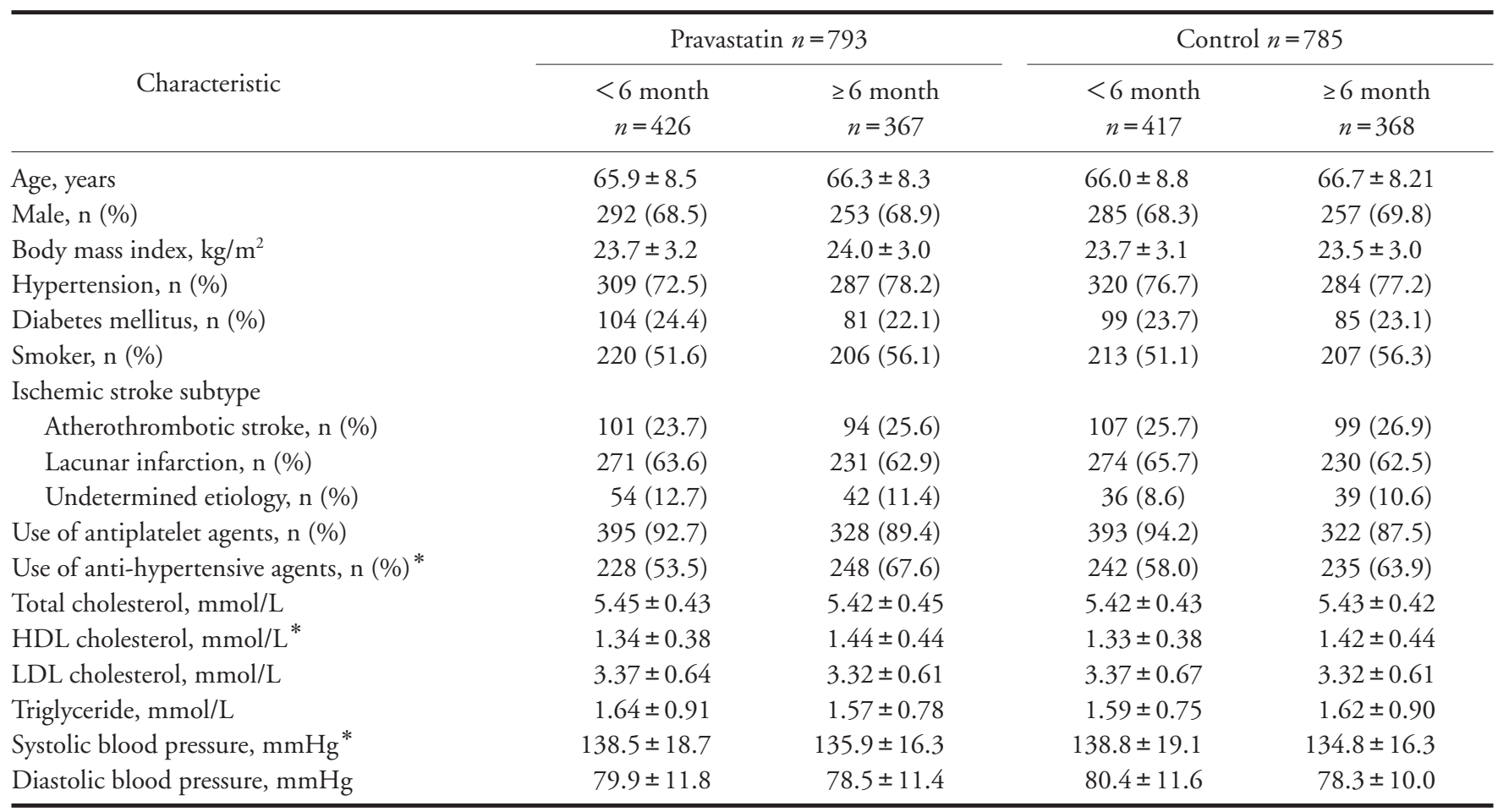

HDL, high-density lipoprotein; LDL, low-density lipoprotein. ${ }_{p}^{*}<0.05$.

main results of the J-STARS study showed that although the rates of total stroke and transient ischemic attack (TIA) in patients treated with pravastatin were similar to untreated individuals, the incidence of atherothrombotic stroke was lower ${ }^{6}$. The Stroke Prevention by Aggressive Reduction in Cholesterol Levels (SPARCL) trial showed that atorvastatin treatment was associated with a $16 \%$ reduction in the risk of recurrent stroke ${ }^{7)}$. The subjects in SPARCL trial were enrolled within 6 months after stroke onset. In general, there is a frequent stroke recurrence within the first year of the initial stroke event. In the J-STARS study patients were recruited for up to 3 years after the initial stroke onset. We hypothesized that the timing of statin treatment may affect its ability to prevent recurrent strokes. In the present study, we evaluated differences of early (within 6 months) or late (after 6 months) pravastatin treatment benefits on the incidence of stroke or TIA, as well as atherothrombotic

Address for correspondence: Naohisa Hosomi, Department of Clinical Neuroscience and Therapeutics, Hiroshima University

Graduate School of Biomedical and Health Sciences, 1-2-3

Kasumi, Minami-ku, Hiroshima 734-8551, Japan

E-mail: nhosomi@hiroshima-u.ac.jp

Received: March 1, 2017

Accepted for publication: July 26, 2017 stroke and other stroke subtypes.

\section{Methods}

\section{Patients}

Details of the rationale, study design, characteristics of the participants, and principal results of the J-STARS study have been published elsewhere ${ }^{6,8,9)}$. Briefly, patients aged 45 to 80 years with a history of non-cardioembolic ischemic stroke within the preceding 1 month to 3 years were enrolled from 123 centers between March 2004 and February 2009. All patients had a total cholesterol level between 4.65 to $6.21 \mathrm{mmol} /$ $\mathrm{L}(180$ to $240 \mathrm{mg} / \mathrm{dL}$ ) at enrollment, without use of statins. The major exclusion criteria included cerebral infarction of determined rare etiology, infarction associated with catheterization or surgery, and preferred use of statins for the treatment of co-morbid coronary artery disease. This study was conducted under the health insurance system of Japan, in accordance with the Declaration of Helsinki and the Ethical Guidelines on Clinical Studies of the Ministry of Health, Labor, and Welfare of Japan. This clinical trial is registered at ClinicalTrials.gov with the number NCT00221104. 
Table 2. Incidence of primary or secondary outcomes

\begin{tabular}{|c|c|c|c|c|}
\hline \multirow[b]{2}{*}{ Incidence of each outcome } & \multicolumn{2}{|c|}{ Pravastatin $n=793$} & \multicolumn{2}{|c|}{ Control $n=785$} \\
\hline & $\begin{array}{c}<6 \text { month } \\
n=426\end{array}$ & $\begin{array}{c}\geq 6 \text { month } \\
n=367\end{array}$ & $\begin{array}{c}<6 \text { month } \\
n=417\end{array}$ & $\begin{array}{c}\geq 6 \text { month } \\
n=368\end{array}$ \\
\hline \multicolumn{5}{|l|}{ Primary outcome } \\
\hline Stroke and TIA, n (\%) & $53(12.4)$ & $39(10.6)$ & $52(12.5)$ & $44(12.0)$ \\
\hline Incidence, 100 person-year & 2.74 & 2.36 & 2.70 & 2.60 \\
\hline \multicolumn{5}{|l|}{ Secondary outcomes } \\
\hline All Strokes, n (\%) & $47(11.0)$ & $38(10.4)$ & $49(11.8)$ & $41(11.1)$ \\
\hline Incidence, 100 person-year & 2.40 & 2.29 & 2.53 & 2.41 \\
\hline Atherothrombotic infarction, $\mathrm{n}(\%)$ & $5(1.2)$ & $3(0.8)$ & $18(4.3)$ & $7(1.9)$ \\
\hline Incidence, 100 person-year & 0.24 & 0.17 & 0.88 & 0.39 \\
\hline Lacunar infarction, n (\%) & $26(6.1)$ & $21(5.7)$ & $20(4.8)$ & $18(4.9)$ \\
\hline Incidence, 100 person-year & 1.29 & 1.23 & 1.00 & 1.03 \\
\hline Cardioembolic infarction, n (\%) & $5(1.2)$ & $2(0.5)$ & $1(0.2)$ & $2(0.5)$ \\
\hline Incidence, 100 person-year & 0.24 & 0.11 & 0.05 & 0.11 \\
\hline Intracranial hemorrhage, $\mathrm{n}(\%)$ & $6(1.4)$ & $6(1.6)$ & $8(1.9)$ & $4(1.1)$ \\
\hline Incidence, 100 person-year & 0.29 & 0.34 & 0.39 & 0.22 \\
\hline TIA, n $(\%)$ & $6(1.4)$ & $2(0.5)$ & $4(1.0)$ & $4(1.1)$ \\
\hline Incidence, 100 person-year & 0.29 & 0.11 & 0.19 & 0.22 \\
\hline Myocardial infarction, n (\%) & $3(0.7)$ & $1(0.3)$ & $2(0.5)$ & $5(1.4)$ \\
\hline Incidence, 100 person-year & 0.14 & 0.06 & 0.10 & 0.28 \\
\hline Vascular accidents, n (\%) & $64(15.0)$ & $49(13.4)$ & $67(16.1)$ & $65(17.7)$ \\
\hline Incidence, 100 person-year & 3.37 & 3.01 & 3.58 & 3.95 \\
\hline Death, n $(\%)$ & $24(5.6)$ & $19(5.2)$ & $19(4.6)$ & $16(4.3)$ \\
\hline Incidence, 100 person-year & 1.14 & 1.08 & 0.92 & 0.89 \\
\hline Hospitalization, n (\%) & $128(30.0)$ & $107(29.2)$ & $113(27.1)$ & $119(32.3)$ \\
\hline Incidence, 100 person-year & 7.38 & 7.30 & 6.55 & 7.84 \\
\hline
\end{tabular}

TIA, transient ischemic attack

\section{Procedures}

Patients were enrolled via a web-based registration and follow-up system provided by the data center, which automatically judged eligibility of each patient and randomly assigned them to pravastatin $(10 \mathrm{mg} /$ day) or control group (1:1 allocation). In the randomization process, prevalence rates of stroke subtype (atherothrombotic stroke), high blood pressure (BP) $(\geq 150$ / $90 \mathrm{mmHg}$ ), and comorbidity of diabetes were dynamically balanced between the two groups. In the pravastatin group, the administration was initiated within 1 month after randomization, and the treatment was continued until final observation. Diet and exercise therapies were reinforced when the total cholesterol levels consistently exceeded $6.21 \mathrm{mmol} / \mathrm{L}(240 \mathrm{mg} / \mathrm{dL})$ at routine clinical visits. Increase of pravastatin dose or addition of other non-statin drugs was allowed only when such reinforcements were insufficient, based on the decision of the primary physician. Use of statins other than pravastatin was prohibited. In the control group, administration of any statin was prohibited.
After randomization, patients were followed up at 2 and 6 months, and annually after that until the study completion. When patients had recurrent stroke or other vascular events, such event information was sent to the data center. Treatment compliance was monitored at every clinical visit.

The primary endpoint was the onset of stroke and TIA. Secondary endpoints contained the onset of each stroke subtype, myocardial infarction, vascular accident, death, and hospitalization. The diagnostic criteria for each stroke subtype, TIA, myocardial infarction, and vascular accidents were shown in previous reports ${ }^{6,8,10)}$. Particularly, stroke and other vascular events were adjudicated by the central event evaluation committees. For the adjudication of events, the final decision was made by consensus of all committee members. Additionally, correctness of all event adjudication was verified by a local neurologist or cardiologist in each participating center, by collating it with the patient medical records. 
Table 3. The Cox proportional hazard model was used to estimate the hazard ratio (HR) and the $95 \%$ confidence interval (CI) for early cohort or late cohort.

\begin{tabular}{llc}
\hline \multicolumn{1}{c}{ Incidence of each outcome } & $\begin{array}{c}\text { Early cohort } \\
<6 \text { month }\end{array}$ & $\begin{array}{c}\text { Late cohort } \\
\geq 6 \text { month }\end{array}$ \\
\hline $\begin{array}{l}\text { Primary outcomes } \\
\text { Stroke and TIA, HR }(95 \% \mathrm{CI})\end{array}$ & $1.02(0.70-1.50)$ & $0.90(0.59-1.39)$ \\
\hline Secondary outcomes & & \\
$\quad$ All Strokes, HR $(95 \% \mathrm{CI})$ & $0.96(0.64-1.43)$ & $0.95(0.61-1.48)$ \\
$\quad$ Atherothrombotic infarction, HR (95\%CI) & $0.30(0.11-0.80)$ & $0.49(0.13-1.90)$ \\
$\quad$ Lacunar infarction, HR $(95 \% \mathrm{CI})$ & $1.28(0.72-2.30)$ & $1.17(0.62-2.20)$ \\
$\quad$ Cardioembolic infarction, HR $(95 \% \mathrm{CI})$ & $5.26(0.61-45.06)$ & $1.00(0.14-7.08)$ \\
$\quad$ Intracranial hemorrhage, HR $(95 \% \mathrm{CI})$ & $0.73(0.25-2.11)$ & $1.53(0.43-5.44)$ \\
TIA, HR (95\%CI) & $1.41(0.42-5.21)$ & $0.51(0.09-2.79)$ \\
\hline
\end{tabular}

Adjusted for the stratification factors at randomization: i.e., stroke subtype at baseline (atherothrombotic stroke vs. others), high BP ( $\geq 150 / 90 \mathrm{mmHg}$ vs. not), and diabetes mellitus (absence vs. presence)

TIA, transient ischemic attack

\section{Statistical Analysis}

In the current post-hoc analyses, we evaluated whether early (within 6 months) or late (after 6 months) pravastatin treatment had differential efficacy with respect to the incidence of atherothrombotic stroke, which was significantly reduced with pravastatin treatment in the J-STARS study ${ }^{6}$, as well as with regard to stroke and TIA, and other stroke subtypes. The data were expressed as the mean \pm standard deviation for continuous variables and as frequencies and percentages for discrete variables. Comparisons of the clinical characteristics were made using analysis of variance (for continuous variables) or $\chi^{2}$ tests (for discrete variables). In accordance with the intention-to-treat (ITT) principle, the efficacy analysis set was defined as the ITT population, and included all randomized patients. The cumulative incidences of time to the first event were estimated by the Kaplan-Meier method. The cumulative incidence rates were analyzed using the person-year method. The cumulative incidence curves for the two groups were compared using a logrank test adjusted for the stratification factors at randomization: i.e., stroke subtype at baseline (atherothrombotic stroke vs. others), high $\mathrm{BP}(\geq 150 / 90 \mathrm{mmHg}$ vs. not), and diabetes mellitus (absence vs. presence). The Cox proportional hazard model was used to estimate the hazard ratio (HR) and the 95\% confidence interval (CI) by adjusting such stratification factors. All analyses were conducted using SAS version 9.3 (Cary, NC, USA). The level of significance was set at $p<0.05$ (2-tailed).

\section{Results}

The 1578 patients were randomly allocated to the pravastatin or control group (793 vs. 785 subjects). There were 426 subjects enrolled within 6 months after stroke onset and 367 subjects enrolled later than 6 months in the pravastatin group. In the control group, 417 subjects were enrolled within 6 months after stroke onset and 368 subjects were enrolled later than 6 months. The baseline characteristics of subjects are shown in Table 1; there were no significant differences in variables among the four groups, except use of anti-hypertensive agents, high-density lipoprotein (HDL) cholesterol, and systolic BP. Specifically, use of anti-hypertensive agents was less, HDL cholesterol was lower, and systolic BP was higher in groups enrolled within 6 months than later than 6 months $(p<0.001$, $p<0.001$, and $p=0.003$ respectively).

The incidence of stroke and TIA, and each stroke subtype are shown in Table 2. The incidence of stroke and TIA was similar in the early cohort and in the late cohort ( 2.48 vs. $2.78 \% /$ year, $p=0.42$ ). The incidence of atherothrombotic stroke was significantly higher in the early cohort compared with the late cohort among control patients ( 0.88 vs. $0.39 \% /$ year, $p=0.049)$. The incidences of any other stroke subtypes were similar in the early cohort and in the late cohort among patients with pravastatin.

The HR of the primary outcome or each stroke subtype using the Cox proportional hazard model is shown in Table 3. Among patients in the early cohort, the incidence of stroke and TIA was similar in pravastatin groups compared with control groups (Fig. 1A; 2.74 vs. $2.70 \% /$ year, $p=0.92$, adjusted HR $1.02[95 \%$ CI 0.70 to 1.50$]$ ). This was also the case among patients in the late cohort in pravastatin groups compared with control groups (Fig. 1A; 2.36 vs. 2.60\%/year, $p=0.64$, adjusted HR 0.90 [95\% CI 0.59 to 1.39 ]). 


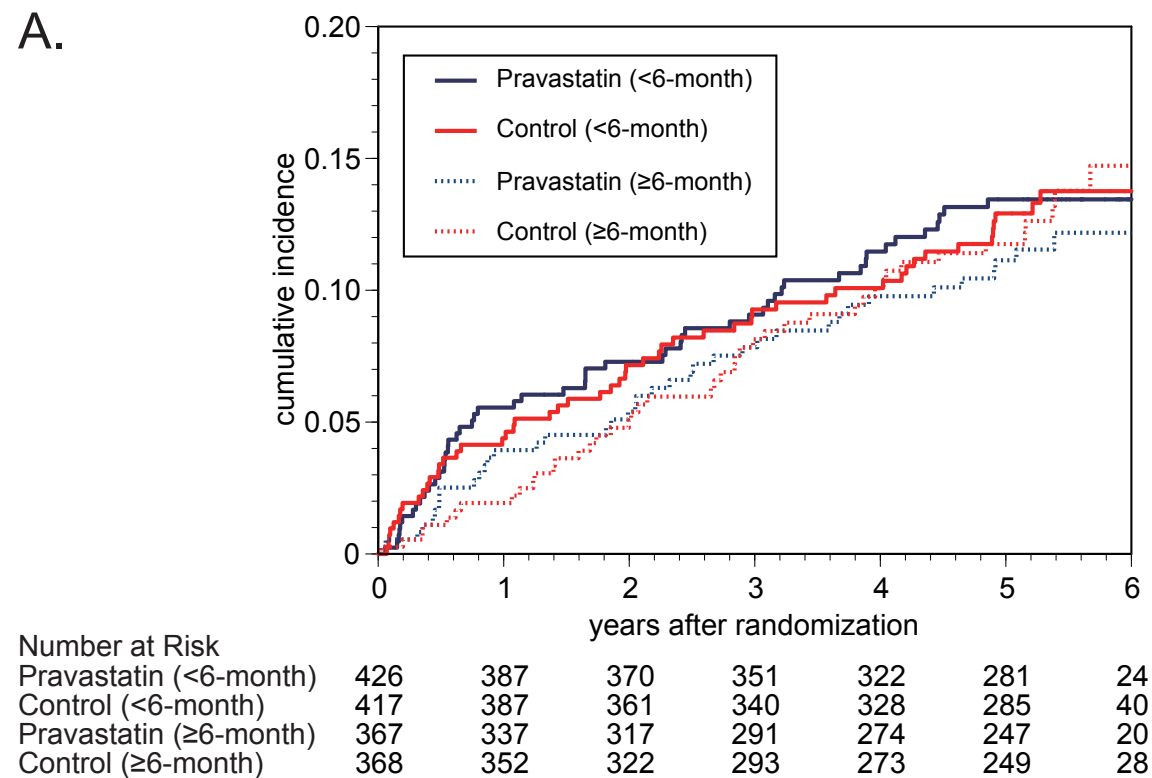

B.

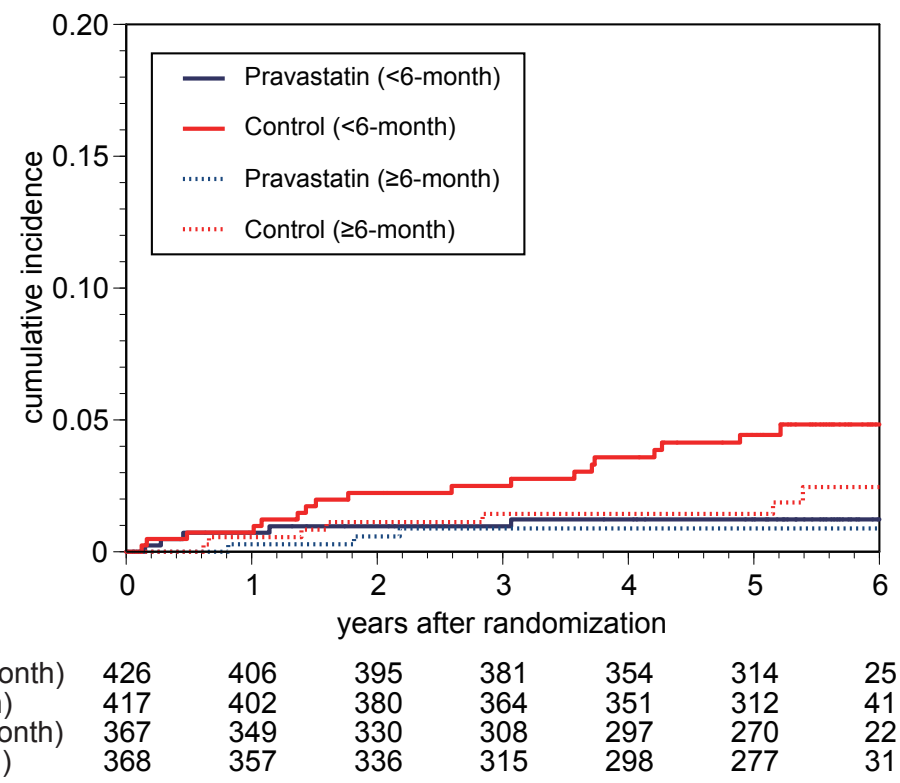

Number at Risk

$\begin{array}{llllllll}\text { Pravastatin (<6-month) } & 426 & 406 & 395 & 381 & 354 & 314 & 25 \\ \text { Control (<6-month) } & 417 & 402 & 380 & 364 & 351 & 312 & 41 \\ \text { Pravastatin ( } \geq 6 \text {-month) } & 367 & 349 & 330 & 308 & 297 & 270 & 22\end{array}$

$\begin{array}{llllllll}\text { Control ( } \geq 6 \text {-month) } & 368 & 357 & 336 & 315 & 298 & 277 & 31\end{array}$

Fig. 1. Kaplan-Meier curves for stroke and transient ischemic attack (A) and atherothrombotic stroke (B) in subjects enrolled within 6 months and later than 6 months from both pravastatin and control groups. In subjects enrolled within 6 months of stroke onset, the incidence of atherothrombotic stroke was significantly lower in the pravastatin group compared with that in the control group (B).

By contrast, the incidence of atherothrombotic stroke was significantly lower in the pravastatin group compared with the control group among patients in the early cohort, (Fig. 1B; 0.24 vs. $0.88 \% /$ year, $p=0.011$, adjusted HR 0.30 [95\% CI 0.11 to 0.80$]$ ]). Among patients in the late cohort, the incidence of atherothrombotic stroke was not significantly reduced by pravastatin (Fig. $2 \mathbf{B} ; 0.17$ vs. $0.39 \% /$ year, $p=0.29$, adjusted HR 0.49 [95\% CI 0.13 to 1.90$]$ ). However, this difference of pravastatin effect on atherothrombotic stroke was not significantly interacted by the early or late cohort $(p=0.59)$. As for the occurrence of lacunar stroke, cardioembolic stroke, and intracranial hemorrhage, no significant differences were observed 
between the control groups enrolled within or later than 6 months after stroke onset. This was also the case for comparisons between pravastatin and control groups. Considering low follow-up rates at 6 year, even though analyzing the HR of primary outcome or each stroke subtype for early cohort or late cohort during 5 year observation, the similar results were obtained (data not shown).

\section{Discussion}

The incidence of stroke and TIA was similar between pravastatin and control groups, regardless of whether enrollment occurred within or later than 6 months after the initial stroke event. However, the incidence of atherothrombotic stroke was more frequent in control subjects enrolled within 6 months of stroke onset. Treatment with pravastatin significantly reduced the incidence of atherothrombotic stroke if given within 6 months of the initial stroke.

In comparison with the baseline characteristics of the SPARCL triall), the parameters related to age and proportion of males, hypertension, diabetes mellitus, and subtype of small vessel disease were higher, whereas that of body mass index was lower, in our J-STARS population. In particular, the times from onset to enrollment were longer in the J-STARS study than the SPARCL trial ${ }^{7)}$. For example, the latter study included patients who had stroke or TIA only within 6 months prior to randomization, whereas the J-STARS study included patients with a history of non-cardioembolic ischemic stroke within the preceding 1-36 months. In the present study, we compared the incidence of stroke or TIA and stroke subtypes between patients enrolled within or later than 6 months after stroke onset. The systolic BP levels of subjects enrolled within 6 months were similar to the baseline levels of patients in the SPARCL trial. On the other hand, HDL cholesterol levels of patients in the J-STARS study were slightly higher than the baseline levels of those in the SPARCL trial. Furthermore, HDL cholesterol levels were lower and systolic BP levels were higher in the subjects enrolled within 6 months of stroke onset compared to those enrolled later than this time. These results suggest that subjects enrolled within 6 months after initial stroke onset were still under insufficient control of low HDL cholesterol and high systolic BP.

As reported in a previous epidemiological study ${ }^{11)}$, there is a frequent stroke recurrence within the first year of the initial stroke event. There were few studies to investigate the recurrent ischemic stroke subtype after non-cardioembolic stroke patients. In the present study, the incidences of lacunar infarction and atherothrombotic infarction were higher than those of other stroke subtypes among control patients. Interestingly, the incidence of atherothrombotic stroke was frequent in the early cohort compared with that in the late cohort among control patients, although the incidence of lacunar infarction was similar in the early cohort and in the late cohort. Our results might indicate that physicians should consider the high incidence of atherothrombotic stroke in early stage after initial noncardioembolic stroke onset. There was no significant difference in the incidence of stroke and TIA between the pravastatin group and control groups enrolled within or later than 6 months. However, the incidence of atherothrombotic stroke was significantly reduced in pravastatin-treated subjects in the early cohort, but not in the late cohort. Although interaction between statin efficacy and the timing of study entry was not significant, our results might suggest that early administration of statin treatment should be considered in order to reduce the risk of atherothrombotic stroke.

The current study has certain limitations. First, this was a sub-analysis of a PROBE designed study. Therefore, it is difficult to lead an assertive conclusion. Second, the small study sample size may not provide the statistical power required to adequately assess the effects of pravastatin with regard to time of enrollment. However, despite this small sample size, a statistically significant reduction of atherothrombotic stroke was obtained in the non-cardioembolic ischemic stroke subjects enrolled within 6 months (early cohort). Third, although there was no significant difference in the incidence rates of stroke and TIA between subjects enrolled within and later than 6 months, the incidence rates of atherothrombotic stroke were significantly lower in subjects enrolled later than 6 months. It may be because those subjects were without atherothrombotic stroke recurrence for 6 months. Finally, we could not evaluate the details of clinical course from the initial stroke onset to the time of study enrollment. Several factors (stroke severity, the management of several medications and so on) might affect that time period of study enrollment. Those factors might also influence the recurrence of stroke.

This post-hoc analysis showed that pravastatin treatment within 6 months of initial stroke onset reduces the incidence of atherothrombotic stroke in Japanese patients with non-cardioembolic stroke. Our findings might suggest a potential efficacy of earlier statin treatment in the prevention of recurrent stroke, although those associations were not conclusive.

\section{Acknowledgments}

The authors thank the patients and their families, and also appreciate study participants, physicians, 
and supporting medical staff and co-workers for their assistance in preparation and execution of this study.

\section{Role of the Funding Source}

This study was initially supported by a grant from the Ministry of Health, Labor and Welfare of Japan. After the governmental support expired, it was conducted in collaboration between Hiroshima University Graduate School of Biomedical and Health Sciences and the Foundation for Biomedical Research and Innovation. The latter organization receives unconditional research grants from several pharmaceutical companies, including DAIICHI SANKYO CO., LTD., which commercializes pravastatin. However, the company was not involved in the design or execution of this study. In addition, the company did not provide pravastatin for this study and has not reviewed the current manuscript.

\section{Disclosures}

KK reports honoraria and grants from Daiichi Sankyo Co., Ltd. HM reports grants from Daiichi Sankyo Co., Ltd. KM reports honoraria from Daiichi Sankyo Co., Ltd. SU reports honoraria from Daiichi Sankyo Co., Ltd. MM reports honoraria and grants from Daiichi Sankyo Co.

The other authors declare no conflicts of interest.

\section{Author's Contributions}

$\mathrm{MM}$ is the principal investigator. NH, YNagai, $\mathrm{KK}, \mathrm{SA}, \mathrm{TN}, \mathrm{KM}, \mathrm{SU}$, and MM are responsible for the conception and design of the study. YNakagawa and TK performed the statistical analysis. NH, YNagai, MN, EM, TY, and MM made data interpretation. $\mathrm{NH}, \mathrm{SA}, \mathrm{TN}$, and $\mathrm{MM}$ designed the figures. $\mathrm{NH}$, YNagai, KK, SA, TN, HM, KM, SU, and MM contributed to draft the report. All authors participated in the finalization of the report.

\section{References}

1) Zhong C, Xia W, Zhong X, Xu T, Li H, Zhang M, Wang A, Xu T, Sun Y and Zhang Y: Lipid Accumulation Product and Hypertension Related to Stroke: a 9.2-Year Prospective Study Among Mongolians in China. J Atheroscler Thromb, 2016; 23: 830-838

2) Matsushima H, Hosomi N, Hara N, Yoshimoto T, Neshige S, Kono R, Himeno T, Takeshima S, Takamatsu K, Shimoe Y, Ota T, Maruyama H, Ohtsuki T, Kuriyama M and Matsumoto M: Ability of the Ankle Brachial Index and Brachial-Ankle Pulse Wave Velocity to Predict the 3-Month
Outcome in Patients with Non-Cardioembolic Stroke. J Atheroscler Thromb, 2017; in press:

3) Morris DR, Ayabe K, Inoue T, Sakai N, Bulbulia R, Halliday $\mathrm{A}$ and Goto S: Evidence-Based Carotid Interventions for Stroke Prevention: State-of-the-art Review. J Atheroscler Thromb, 2017; 24: 373-387

4) Tada H, Kawashiri MA, Nohara A, Inazu A, Kobayashi J, Yasuda K, Mabuchi H, Yamagishi M and Hayashi K: Lipid Management in a Japanese Community: Attainment Rate of Target Set by the Japan Atherosclerosis Society Guidelines for the Prevention of Atherosclerotic Cardiovascular Diseases 2012. J Atheroscler Thromb, 2017; 24: 338-345

5) Usui T, Nagata M, Hata J, Mukai N, Hirakawa Y, Yoshida D, Kishimoto H, Kitazono T, Kiyohara Y and Ninomiya T: Serum Non-High-Density Lipoprotein Cholesterol and Risk of Cardiovascular Disease in Community Dwellers with Chronic Kidney Disease: the Hisayama Study. J Atheroscler Thromb, 2017; 24: 706-715

6) Hosomi N, Nagai Y, Kohriyama T, Ohtsuki T, Aoki S, Nezu T, Maruyama H, Sunami N, Yokota C, Kitagawa K, Terayama Y, Takagi M, Ibayashi S, Nakamura M, Origasa H, Fukushima M, Mori E, Minematsu K, Uchiyama S, Shinohara Y, Yamaguchi T, Matsumoto M and collaborators JS: The Japan Statin Treatment Against Recurrent Stroke (J-STARS): A multicenter, randomized, open-label, parallel-group study. EBioMedicine, 2015; 2: 1071-1078

7) Amarenco P, Bogousslavsky J, Callahan A, 3rd, Goldstein LB, Hennerici M, Rudolph AE, Sillesen H, Simunovic L, Szarek M, Welch KM and Zivin JA: High-dose atorvastatin after stroke or transient ischemic attack. N Engl J Med, 2006; 355: 549-559

8) Nagai $Y$, Kohriyama T, Origasa H, Minematsu K, Yokota C, Uchiyama S, Ibayashi S, Terayama Y, Takagi M, Kitagawa K, Nomura E, Hosomi N, Ohtsuki T, Yamawaki T, Matsubara Y, Nakamura M, Yamasaki Y, Mori E, Fukushima M, Kobayashi S, Shinohara Y, Yamaguchi T, Matsumoto $\mathrm{M}$ and Investigators J-S: Rationale, design, and baseline features of a randomized controlled trial to assess the effects of statin for the secondary prevention of stroke: the Japan Statin Treatment Against Recurrent Stroke (JSTARS). Int J Stroke, 2014; 9: 232-239

9) Nakamura M, Fukukawa T, Kitagawa K, Nagai Y, Hosomi N, Matsumoto M, Minematsu K, Uchiyama S and Miyamoto Y: ANNALS EXPRESS: Ten-year standardization of lipids and high-sensitivity C-reactive protein in a randomized controlled trial to assess effects of statins on secondary stroke prevention: Japan Statin Treatment Against Recurrent Stroke (J-STARS). Ann Clin Biochem, 2017; 4563217693651

10) Kitagawa K, Hosomi N, Nagai $Y$, Kagimura T, Ohtsuki T, Origasa $H$, Minematsu K, Uchiyama S, Nakamura M and Matsumoto M: Reduction in high-sensitivity C-reactive protein levels in patients with ischemic stroke by statin treatment: Hs-CRP Sub-study in J-STARS. J Atheroscler Thromb, 2017; in press:

11) Hata J, Tanizaki Y, Kiyohara Y, Kato I, Kubo M, Tanaka K, Okubo K, Nakamura H, Oishi Y, Ibayashi S and Iida $\mathrm{M}$ : Ten year recurrence after first ever stroke in a Japanese community: the Hisayama study. J Neurol Neurosurg Psychiatry, 2005; 76: 368-372 